AperTO - Archivio Istituzionale Open Access dell'Università di Torino

The orbital: a pivotal concept in the relationship between chemistry and physics? A comment to the work by Fortin and coauthors

This is the author's manuscript

Original Citation:

Availability:

This version is available http://hdl.handle.net/2318/1659617

since 2019-01-30T14:45:01Z

Published version:

DOI:10.1007/s10698-017-9293-4

Terms of use:

Open Access

Anyone can freely access the full text of works made available as "Open Access". Works made available under a Creative Commons license can be used according to the terms and conditions of said license. Use of all other works requires consent of the right holder (author or publisher) if not exempted from copyright protection by the applicable law. 


\title{
The orbital: a pivotal concept in the relationship between chemistry and physics? A comment to the work by Fortin and coauthors
}

\author{
Giovanni Villani ${ }^{1 *}$, Elena Ghibaudi ${ }^{2}$, Luigi Cerruti $^{3}$
}

1 - Istituto di Chimica dei Composti OrganoMetallici, ICCOM-CNR (UOS Pisa), Via G. Moruzzi 1, I-56124 Pisa, Italy

2 - Dept. of Chemistry, University of Torino, Via Giuria 7, I-10125 Torino, Italy

3 - Independent researcher, Viale delle acacie 12, I-10148 Vinovo (TO), Italy

* Author to whom correspondence should be addressed; E-mail: villani@ pi.iccom.cnr.it

\begin{abstract}
The present work is a comment of a recent paper by Fortin and coauthors (Fortin 2017) in which the authors propose the introduction of Bohmian mechanics (BM) in the philosophy of chemistry and the use of standard quantum mechanics (SQM) as a mere instrument of prediction. This way would allow overcoming the obstacles found in linking molecular chemistry and quantum mechanics. Starting from some remarks on the orbital concept, we highlight and discuss some general issues that need to be taken into account when two scientific theories coexist within the same investigation field, i.e. SQM and BM.
\end{abstract}

Keywords

Bohmian mechanics; Stadard Quantum Mechanics; orbital concept; Quantum electron state; underdetermination; pragmatic view of theories.

\section{Commentary}

The work by Fortin, Lombardi and Martinez Gonzales is extremely relevant and raises some general issues that deserve to be underlined. The authors propose that the introduction of Bohmian Mechanics (BM) in the philosophy of chemistry might overcome, or at least decrease, the obstacles found in linking molecular chemistry and quantum mechanics: "At this point, it is worth clearly stressing that the arguments presented above belong to the field of the foundations and the philosophy of chemistry [...] the conceptual difficulties derived from the link between molecular chemistry and quantum mechanics would be tackled from a Bohmian perspective" (Fortin 2017).

Nevertheless, the authors neither think nor propose to replace standard quantum mechanics (SQM) with BM in scientific practice: "By no means is it suggested that SQM should be replaced with BM in the actual practice of molecular chemistry or of quantum chemistry. In fact, the use of BM not 
only is unnecessary in a field whose practitioners are taught and trained with the formal resources of SQM, but also is not convenient to the extent that the mathematical calculations of BM rapidly become highly intractable with the increase of the system's complexity". They suggest using SQM as "a mere instrumental tool for prediction" (Fortin 2017).

This would be possible as BM and SQM are distinct theoretical tools, sharing a same empirical content: "Bohmian mechanics (BM) is not an interpretation of the standard formalism, but a different theory with the same predictive power as SQM. Both theories are empirically equivalent (Goldstein 2016)". This leads to underdetermination: "The possibility of empirical equivalence between scientific theories leads to the thesis of the underdetermination of scientific theory by evidence (or by data), which is based on the simple idea that the evidence available to us may be insufficient to determine what beliefs we should hold in response to it".

This Comment is structured as follows: in the first part, we will highlight and discuss some general issues that need to be taken into account when two scientific theories coexist within a same investigation field, i.e. SQM and BM. We will then analyze the examples that Fortin and coauthors have chosen for showing that the description provided by SQM is totally different from that provided by molecular chemistry. Fortin and coauthors discuss three cases: (a) the notion of orbital, (b) the components of the atom and (3) the concept of molecular structure. We will limit our discussion to the first one. A discussion of the concept of orbital cannot neglect the essential aspect of modelling. Hence, the notion of scientific model will be briefly discussed and related with the concept of orbital. A short conclusion will be finally provided.

\section{Two scientific theories for the same empirical field}

The paper by Fortin and coauthors draws attention to the epistemic relationship between theories. This relationship is discussed for two distinct cases: theories belonging to different disciplines (chemistry vs. physics) and rival theories within a same discipline (SQM vs. BM). These are totally different cases that Fortin and coauthors tackle differently, as they actually deserve. Nonetheless, their discussion should be preceded by a general evaluation of the epistemic nature of theories. In fact, such an evaluation provides a general philosophic frame for the more specific issue of the relationship between distinct theories. In addition, it helps to highlight aspects of both theories which are relevant to an epistemic analysis of their connection. The viewpoint from which theories are considered is important because the "choice of a view of theories is a form of philosophical modeling of science" (Griesemer 2013). Historically, three standpoints on scientific theories have been established. The syntactic view may be related to the 'logical positivists' of the Vienna Circle and the subsequent elaboration by Carl Hempel in the 1960s. It considers a scientific theory as a set of sentences that may be expressed in the appropriate logical language and derived from a metamathematical axiomatization of the theory. In a sense, the very same title of the foundational work by Rudolf Carnap (Der logische Aufbau der Welt, 1928) expresses very clearly the cognitive purposes of the proponents of this viewpoint. The semantic view shifts the philosopher's attention from the logical analysis of theories towards an investigation based on mathematical models. A relevant supporter of this standpoint is the Dutch philosopher Bas van Fraassen (mentioned by Fortin and coauthors). In his 1980 essential work, van Fraassen clearly speaks of "failure of the syntactic approach" and strongly claims that: "the notions of truth and model belong to semantics" (van Fraassen 1980, pp. 53 and 43). According to the Dutch philosopher "Any structure which 
satisfies the axioms of a theory [...] is called a model of that theory", and "The models occupy centre stage". Van Fraassen's models are to be intended in the strictly mathematical sense of the theory of models. For example, talking about the Bohr's atomic model, he distances himself from this use of the term: "in the scientists' use, 'model' denotes what I would call a model-type" (van Fraassen 1980, p. 44). In synthesis: "For the Syntactic View, what is not (or cannot be) reconstructed axiomatically is not theoretical, while for the Semantic View, what is not (or cannot be) modeled mathematically is not theoretical". Based on this sharp contrast, it is not surprising that the supporters of the syntactic vs. semantic views have often chosen a "strategy of combat" within the philosophical debate (Winther 2015).

A more ecumenical strategy characterizes the supporters of a pragmatic view of scientific theories. The pragmatic view does not deny the effectiveness of logic-linguistic analysis and mathematical models; yet, it considers that the scientific enterprise relies on a wide spectrum of cognitive tools that includes non formal tools such as analogies, natural kinds, exemplars (in Kuhn's acceptation). As regards models used by (real) science, the pragmatic view observes that there is "a variety of modeling syntaxes - mathematics, diagrams, narratives, simulations, and programs" (Winther 2012), that need to be discerned and made coherent, "theoretical models (possible realizations of theories) [and] empirical models of phenomena (representations of possible realizations of phenomena)" (Griesemer 2013).

Clearly, the choice of a specific philosophical position on the structure of theories is not neutral with respect to the focus of the epistemic investigation. Let's tackle the parallel between chemistry and physics proposed by Fortin and coauthors. In the discussion of the concept of orbital that will be presented in the next section, we will see that the pragmatic view draws the attention to the orbital models, with their inherent (formal and non formal) assumptions. In our view, this is a more effective analysis as compared to the analysis fostered by a syntactic view, which would rather investigate a possible axiomatic deduction of orbitals from the foundational principles of SQM and/or BM. Seemingly, the pragmatic view is more convincing than the semantic view, which describes orbitals as mere mathematical structures.

As regards the comparison between SQM and BM, Fortin and coauthors raise two problems that should be kept apart: the underdetermination and the empirical equivalence of theories. Concerning underdetermination, Fortin's approach does not consider that there are at least two kinds of underdetermination: holistic and contrastive. According to the former, any theory is a web of belief (Quine 1970); hence it is underdetermined with respect to the experimental basis. Conversely, the latter takes into account a given experimental basis and maintains that several theories may be equally supported by that very same basis (Stanford 2016). The comparison between SQM and BM, proposed by Fortin and coworkers, comes under the contrastive underdetermination. The hesitation on the foundational proposal by Fortin is reinforced by the fact that even the empirical equivalence between SQM and BM is called into question. It is "controversial because it is not clear that we understand Quantum Mechanics well enough to say convincingly what formulations of it count as genuinely different theories" (Stanford 2001). In addition, even amongst theoretical physicists who admit the empirical equivalence of theories, the landscape of quantic theories is more varied than the simple comparison between SQM and BM. James Hartle assumes as testbed for valid quantummechanical formulations "a formulation that [...] reproduces the standard textbook answers for the probabilities of measurements". Hartle counts 13 distinct formulations as valid, although he finally compares only three of them: SQM, BM and his own formulation that "is logically consistent, 
consistent with experiment as far as is known, consistent with textbook predictions for measurements, and applicable to the most general physical systems". Just like Fortin and coworkers, Hartle also admits that - due to the empirical equivalence - the lab cannot be the discriminant for establishing which theory is to be preferred. He reports: "We may be able to distinguish interpretations by their utility and/or their promise as starting points for generalizations or alternatives to quantum theory" (Hartle 2005). It is worth recalling that, the experimental basis being open, there isn't any guarantee that the experimental equivalence between SQM and BM is permanent (Stanford 2016). On the other hand, Hartle's use of an extra-theoretical and pragmatic word such as 'utility' seems an explicit reminder of the opportunity of a pragmatic view of theories.

\section{The examples proposed by Fortin and coauthors}

Fortin and coworkers propose three examples aimed at discussing the differences between SQM and molecular chemistry. The three of them share a common issue: SQM does not allow identifying nor fixing the position of a particle (whatever the particle is, a generic atomic component, an electron, the atomic nucleus). Conversely, this is systematically done in quantum chemistry. We will comment only one example out of three, i.e. the orbital. In fact, all of them deserve comments; particularly the concept of molecular structure, which is probably the most relevant as it deals with the chemical approach to living systems (a topic that has been tackled by one of us, Villani 2017). Unfortunately, this is a much too wide topic to be treated in a short comment like this; a more detailed analysis will be proposed in the future.

The problem of clarifying the concept of orbital has been widely treated in the literature, as witnessed by the essential bibliography provided by Fortin and coworkers. They claim that, in molecular chemistry, orbitals are conceived as space regions whereas in quantum mechanics they are mathematical functions. According to Fortin and coworkers, this is a main difference between the two scientific areas. The concept of orbital cannot be exhaustively tackled nor discussed in a commentary. Nevertheless, we would like to underline some aspects of the problem, in line with Fortin's viewpoint that BM and SQM should coexhist and be linked to each other.

The authors write: “'Orbital' is a term that is used both in chemistry and in physics, but refer to different items in the two disciplines (Scerri 2000; Labarca and Lombardi 2010; Mulder 2011). According to the Oxford Dictionary of Chemistry, both the wave function and its corresponding spatial region of high electron density can be called 'orbital' (Daintith 2004)". Fortin and coauthors claim that the right definition of orbital is a mathematical function, although this concept is substantialized by chemists: "Nevertheless, from referring a mathematical entity, the concept of orbital is rapidly substantialized, and the talk of 'the shape of an orbital' enters the scene" (Fortin 2017). Strangely, they mention Mulder (2011) without apparently realizing that he says: "Orbitals are states, not entities", although a few lines later Mulder points out: "So far I have taken orbitals to be one-electron wave functions". Anyway, later in the paper, Fortin and coworkers report: "Let us consider the example of the hydrogen atom. According to SQM, when the atom is in the ground state, the electron is in the state $1 \mathrm{~s} "$.

These very same quotes suggest that there is a third possibility for the concept of orbital, distinct from both the mathematical function and the space region: an orbital is a scientific concept that, within SQM and a specific model identifies the electron state. Whenever we say that - in an electronic spectroscopy experiment - a beam of a given wavelength allows the electron within the hydrogen atom to switch from the 1 s ground state to the 2 s excited state, we do not mean to say that 
the electron switches from a mathematical function to another, nor we mean to say that it moves from one space region to another. We mean to say that that the electron switches from one state to another.

Two clarifications are needed. First of all, the state of the electron we are dealing with is a dynamic state. Nevertheless, both the mathematical function $(\Psi)$ associated with the electron state (that allows calculating specific electronic properties such as the probability of localization within a space region) and the space region some time associated to it are essentially static. As a matter of fact, the definition of quantum electron state includes dynamical features (such as angular momenta), but these are identified by numbers and not by a time-dependent function, just like its static features. This occurs because - although quantum mechanics starts with the time-dependent Schrödinger equation - it is quickly replaced by the time-independent equation, which leads to the determination of stationary states whose time dependence is solely associated with phase Exp[-iEt]. Whenever a state perturbation occurs, these states are no longer stationary and their dynamic aspects come out. For example, in the presence of an electric field, a molecule is polarized, hence it modifies its electron density distribution (from the classical viewpoint, an electronic charge flow occurs inside the molecule).

In the second place, the electron state is fully expressed by the spin-orbital, where the spin component is defined as well; hence the expression that 'an orbital can be occupied by two electrons'. This expression deserves caution. If the electron is a mathematical function, how can this expression be possibly interpreted? Can a mathematical function 'be occupied' by something? And, in general, what do we mean whenever we say that an electron 'is' in the 1s or $2 p$ orbital? Actually, we mean that both the static and dynamic electron features show specific symmetry properties: e.g., the probability distribution of finding an electron at a distance $r$ from the nucleus is spherical whenever the electron is in a $s$-state. This is equally true for actual electron states and virtual states, that exist only as a possibility. The latter represent the possibilities that the electron properties change in a specific way when the electron is excited and shifts from one state to the other. Moreover, it has to be stressed that, from a didactic viewpoint, the representation of orbitals as either empty (virtual) or full (real) boxes generates misconceptions and are misleading.

Clearly, this is well known to Fortin and coworkers as well as to any chemist, although discussions on the possibility of 'seeing' orbitals leave us doubtful to some extent. Therefore, what is the point of persisting in identifying a scientific concept with a mathematical function or a space region? In addition, this is very confusing for students that need to learn the correct concept, thus avoiding to materialize the orbital or to take it as a purely formal and somehow arbitrary concept.

Getting back to the paper by Fortin and coworkers, it seems to us that the orbital - taken as a scientific concept that identifies the electron state and allows determining its properties - is essential in both SQM and BM. In fact, mechanical theories need to be compared in the wider perspective of the discussion of the electron state. In classical mechanics, the dynamic state of a particle is represented by a point in the phase space and it is identified by a generalized coordinate and by its conjugate momentum. With Bohr, the electron already lost its punctual feature and became an orbit. Subsequently, SQM 'spread out' the electron state over the whole space, albeit in a non uniform way. It is precisely this lack of homogeneity that allows visualizing the space regions associated with a specific electron state (through an act that is a mere representation and not a materialization of the orbital). In BM, each particle is classical and is associated with a wave that drives its movement (pilot-wave) and generates a quantum potential over the particle. It seems to us 
that there has been a lack of reflection as regards how to define the particle state in the presence of such a non-local, holistic potential.

As the last point of this analysis, we would like to discuss another crucial aspect that - in our view is insufficiently highlighted in the orbital description of multi-electronic atomic and molecular states. In general, orbitals are said to represent the state of the electron in a hydrogen atom (hence the term hydrogenoid). This is not fully correct. Strictly speaking, orbitals represent electron states within a system made of one electron and an atomic nucleus, both of which are considered as material points, the only relevant force is the Coulombian one (the others are taken as negligible) and there are no relativistic effects. Since this system is similar to the hydrogen atom, the model system is almost always confused with the physical system. As a result, orbitals are considered as states of a physical system instead of being part of a model. Whenever the modellistic aspect of the orbital description is wiped out - both in the hydrogen atom and in multi-electron atoms and molecules - the rationale behind such a description becomes unclear. In fact, one can no longer understand why the electron states in a hydrogen atom can be useful to describe the states of completely distinct systems, such as multi-electron atoms; as a consequence, disputes about the degree of 'reality' of an orbital come out. According to Mulder, quoted by Fortin and coauthors: "The case of electrons in many-electron atoms and molecules is merely quantitatively, not qualitatively, different. Therefore, talking about orbitals in terms of either existing or not is not just, as Ostrovsky sees it, less appropriate than in terms of being a better or worse approximation; it is mistaken. The upshot of this is that I substantially agree with Ostrovsky and Schwarz, although I believe their reflections on existence lead astray. Wave functions that are products of orbitals can be better or worse approximations to the exact wave function of an atom or molecule; and that is all there is to be said concerning the ontological status of orbitals in many-electron systems" (Mulder 2011). Apart from the identification of the orbital with the wave function, we substantially agree with Mulder (and Ostrovsky and Schwarz). Nevertheless, we believe that this quote lacks a term model - that would have made the argument much clearer: a model is always a representation of the physical realm, an abstraction that takes into account a selection of features of that realm. Such representation can be more or less effective; anyway, this has nothing to do with the actual existence of orbitals within the atomic systems. It is just pertinent to the applicability of the model in a new context.

\section{Models in science}

The problem of the materialization of abstract entities, conceived within a well defined theoretical frame, is related with the lack of a clear-cut distinction between the phenomena that need to be interpreted and the interpretative models that may serve this scope.

According to Jean Perrin, modeling means: "Remplacer du visible compliqué par de l'invisible simple" (Jean Perrin cit. in Walliser 1977). Perrin underlines that a model must never be taken as a reproduction of a portion of the physical realm. According to Walliser: "in its wider acceptance, the notion of models refers to any representation of a real system, either abstract or concrete, expressed in a verbal, graphical or mathematical form" (Walliser 1977). Hence, a scientific model is a heuristic tool, a logical construct that may serve to justify a given fact and make prediction on the behavior of a real system. A model being a tool, is never an end in itself; it always supplies answers to problematic questions. As a consequence, a model should never be said to be right or wrong; it should rather be found appropriate/inappropriate for the description of a system or a phenomenon. 
The abstract and interpretive character is particularly evident in the case of orbitals: this concept arises within a theory (SQM) and is a conceptual tool fit for the interpretation of the behavior of a material system. In chemistry, the concept of orbital - intended as a scientific concept that provides information on the electron state within the SQM - allows providing a systematic interpretation for atomic and molecular properties. In addition, it allows calculating the static and dynamic properties of electrons in these systems and the interpretation of spectroscopic data.

The concept of orbital is nothing but a conceptual tool, a model whose justification and field of existence lies within a theory. To materialize and/or mathematize the orbital is an incorrect conceptual operation with several negative outcomes, as it implies mixing up the level of reality with the level of models. This general problem has been successfully tackled by Robert Rosen (Rosen 1985), who developed a meta-epistemological model known as 'modelling relation'. According to Rosen, the process of building up of scientific knowledge implies the definition of the relationship between a natural system (NS), i.e. a portion of the physical realm represented by "a set of qualities, to which definite relations can be imputed" (Rosen 1985) and a formal system (FS) that models it. The FS results from a codification of the NS, based on rules defined by the modeller, consistent with the constraints imposed by empirical/experimental settings and findings. The relationship between FS and NS has also a predictive role towards the latter: "We seek to encode natural systems into formal ones [such that] the inferences or theorems we can elicit within such formal systems become predictions about the natural systems we have encoded into them" (Rosen 1985, p. 74). Whenever the predictions made through the FS find a correspondence in the NS, we say that the FS is a good model of the NS. Observables "are the fundamental units of natural systems, just as percepts are the fundamental units of experience" (Rosen 1991, p. 60). Orbitals are not observables; hence, according to Rosen, they are undoubtedly part of the FS.

It seems relevant to underline that "the act of modeling is really the act of relating two systems in a subjective way" (Schierwagen 2009). Thanks to that, it is possible to model a same NS through distinct FSs. The comparison between the system description provided by SQM and BM falls within this case. The relationship between the two theories can be established by their common reference to a same NS as well as by their reference to common concept, such as the electron state (orbital).

Rosen stresses that "the difficulty and challenge in establishing such relations [between FS and NS] arises from the fact that the entities to be related are fundamentally different in kind. A NS is essentially a bundle of linked qualities, or observables, coded or named by the specific percepts which they generate and by the relations which the mind creates to organise them. As such, a NS is always incompletely known.[...] A FS, on the other hand, is entirely a creation of the mind, possessing no properties beyond which enter into its definitions and their implications" (Rosen 1985). Nevertheless, a FS is not arbitrary: in fact, the NS and FS are connected by a circular relationship, defined by the modelling procedures and the empirical verifications of the effectiveness of the model in the interpretation of specific phenomena.

\section{Conclusions}

In conclusion, we would like to underline that the concept of orbital as an expression of the electron state is crucial and allows comparing distinct theories. We have stressed that orbitals belongs to a modellistic description of the physical realm. Such a conception helps to overcome two conceptual 
attitudes (the materialization and the mathematization of the concept of orbital) that may lead to logical contradictions and generate misconceptions in teaching practice.

The work by Fortin and coauthors provides a relevant contribution to the discussion concerning the relationship between disciplines and theories. Namely, it highlights that the same concept - the orbital - is employed very differently in molecular chemistry and quantum mechanics: for example, the two domains make a very different use of the spatial visualisation of some properties of atomic and molecular systems. This is an important aspect that should not be neglected whenever chemistry and physics are confronted.

Nevertheless, such a confrontation needs to be set in the wider perspective of the investigations on the nature of each discipline. Each of the three above-mentioned views of theories allows us to construct a different model of traditional disciplines (chemistry, physics, biology, etc.). In our opinion, the foundational problems can be tackled in very different ways depending on the object of the epistemological analysis. This object may be a theoretical corpus understood as the result of an axiomatic derivation, a structure of connected mathematical models, or a set of modelling syntaxes. The last case corresponds to the pragmatic view of theories: from this vantage point, no 'external' foundation is seen as necessary for a discipline.

From a pragmatic viewpoint, distinct syntactic models may be included in the theoretical corpus of a discipline; so, the possibility of using both SQM and BM within the theoretical structure of chemistry is plausible. Conversely, the proposal of making a clear-cut distinct use of two theories (a mere instrumental use for SQM and a foundational use for BM) does not seem necessary.

Clearly, the relevance of deepening the discussion of the relationship between different disciplines and theories is undoubted. Had we analysed the example concerning molecular structure, the discussion would have been extended to a third discipline - biology - besides physics and chemistry.

\section{References}

Carnap, R.: Der logische Aufbau der Welt. Weltkreis-Verlag, Berlin (1928)

Daintith, J.: Oxford Dictionary of Chemistry. Oxford University Press, NewYork (2004)

Fortin, S., Lombardi, O., Martınez Gonzalez, J.C.: The relationship between chemistry and physics from the perspective of Bohmian mechanics. Found Chem., 19, 43-59 (2017)

Griesemer, J.: Formalization and the Meaning of Theory in the Inexact Biological Sciences, Biol. Theory, 7, 298-310 (2013)

Hartle, H.B.: What Connects Different Interpretations of Quantum Mechanics?. In: A. C. Elitzur, S. Dolev, N. Kolenda, Quo Vadis Quantum Mechanics? Springer, Berlin (2005), pp. 73-82.

Labarca, M., Lombardi, O.: Why orbitals do not exist? Found. Chem. 12, 149-157 (2010)

Mulder, P.: Are orbitals observable? Hyle-Int. J. Philos. Chem., 17, 24-35 (2011)

Quine, W.V., Ullian J.S.: The Web of Belief. Random House, New York (1970)

Rosen, R.: Anticipatory Systems. Pergamon Press, Oxford (1985) 
Rosen, R.: Life Itself: A Comprehensive Inquiry Into the Nature, Origin, and Fabrication of Life, Columbia University Press, New York (1991)

Scerri, E.: The failure of reduction and how to resist disunity of the sciences in the context of chemical education. Sci. Educ. 9, 405-425 (2000)

Schierwagen, A.: Brain Complexity: Analysis, Models and Limits of Understanding. In: Methods and Models in Artificial and Natural Computation, Springer, Berlin (2009) pp. 195-204.

Stanford, P.K.: Refusing the Devil's Bargain: What Kind of Underdetermination Should We Take Seriously? Phil.Sci., 68, Supplement, Part I, S1-S12 (2001)

Stanford, P.K.: Underdetermination of Scientific Theory. In The Stanford Encyclopedia of Philosophy (2016). URL: https://plato.stanford.edu/entries/scientific-underdetermination/

Van Fraassen, B.: The Scientific Image. Oxford University Press (1980)

Villani, G.: Chemical perspective in the study of living beings: a systemic complexity approach. Found Chem., 19, 77-91 (2017)

Walliser, B.: Système et modèles. Ed. du Seuil, Paris (1977)

Winther, R.G.: Mathematical modeling in biology: philosophy and pragmatics. Front.Plant Sci., 3, $102(2012)$

Winther, R.G.: The Structure of Scientific Theories. In: The Stanford Encyclopedia of Philosophy (2015). URL: https://plato.stanford.edu/entries/structure-scientific-theories/ 